\title{
Research of expression and correlation of ADAM8 and EGFR in the osteosarcoma tissue
}

\author{
Yan Liu*, Jie Wen \\ Department of Orthopaedics, Baogang Hospital, Baotou, Inner Mongolia 014010, China
}

Received: April 12, 2019

Accepted: May 30, 2019

Online Published: June 10, 2019

DOI: $10.5430 /$ dcc.v6n2p1

URL: https://doi.org/10.5430/dcc.v6n2p1

\begin{abstract}
Objective: To lay a foundation for the research on the targeted therapy of osteosarcoma or the regulatory mechanism of the occurrence and development of osteosarcoma by studying the expression of a disintegrin and metalloproteinase domain-containing protein 8 (ADAM8) and epithelial growth factor receptor (EGFR) in osteosarcoma.

Methods: Osteosarcoma tissues, osteochondroma tissues, osteoarthritis tissues and normal bone tissues were selected and included in this research. By use of tissue chips and immunohistochemistry, the expression levels of ADAM8 and EGFR in the previously-mentioned tissues were observed and compared. Spearman's rank correlation test was used to analyze the correlation of ADAM8 and EGFR and their relationship with clinicopathological factors in these cases.

Results: The positive expression levels of ADAM8 in osteosarcoma tissues, osteochondroma tissues, osteoarthritis tissues and normal bone tissues were $80.95 \%, 36.36 \%, 62.86 \%$ and $24.14 \%$ respectively, the difference was of statistical significance $(p<.05)$; the positive expression levels of EGFR in osteosarcoma tissues, osteochondroma tissues, osteoarthritis tissues and normal bone tissues were $66.67 \%, 9.09 \%, 40.00 \%$ and $4.76 \%$ respectively, the difference was of statistical significance $(p<.05)$; Spearman's rank correlation test showed that ADAM8 was positively correlated with EGFR $(r=.379, p<.05)$.

Conclusions: Both ADAM8 and EGFR have a much higher expression in osteosarcoma tissues, and the combined detection of two indexes can be considered as a new method in the clinical diagnosis of osteosarcoma.
\end{abstract}

Key Words: ADAM8, EGFR, Osteosarcoma, Correlation

\section{INTRODUCTION}

Osteosarcoma is a common, rapidly-growing and primary malignant bone tumor. Most of patients are diagnosed as osteosarcoma with high phenotype. Pulmonary metastasis occurs in more than $15 \%$ of patients, and local metastasis happens to above $80 \%$ of patients. Therefore, it is rarely seen that osteosarcoma is cured only depending on surgical operation. The prognosis in young patients with terminal local lesion can be significantly improved by use of multimodal chemotherapy. ${ }^{[1,2]}$ However, patients with metastasis or a failure in adjuvant chemotherapy still show poor prognosis.
Currently, the molecular mechanism of the occurrence and development of osteosarcoma remains unclear. This type of research can be conducive to providing a new theoretical basis for the diagnosis, the prognosis, the clarification of the molecular mechanism and the targeted interventions of osteosarcoma in the clinical application, with a good social benefit. DAM8 (CD156), as a member of ADAM (a disintegrin and metalloproteinase domain-containing protein) family, plays an important role not only in cell adhesion molecules and the matrix tissues of cytokine receptors but also in the diagnosis and prognosis of the disease. ${ }^{[3]}$ Some researches

\footnotetext{
*Correspondence: Yan Liu; Email: nmgbgyyyy@163.com; Address: Department of Orthopaedics, Baogang Hospital, Baotou, Inner Mongolia 014010, China.
} 
have showed that red blood cells which lack ADAM8 can not separate and form within vessel lumens, and they can depress the growth of malignant tumors by inhibiting the expression of ADAM8. Hemandez et al. ${ }^{[4]}$ also have reported that ADAM8 plays a significant role in bone metastasis of lung cancer, and its high expression is positively correlated to the prognosis of lung cancer. In addition, many a scholar has found that the expression of ADAM8 is up-regulated in different types of cancerous tumors. Whereas, the expression of ADAM8, related with high-grade lung adenocarcinoma and non-small cell lung cancer, has been recommended to be considered as a serum marker of lung cancer. Epithelial growth factor receptor (EGFR) is a receptor for cell proliferation and signaling pathway of epithelial growth factors (EGF), and it is a member of ErbB family.

\section{DATA AND METHODS}

\subsection{General information}

Tissue chips from 50 cases of osteosarcoma tissues were purchased, and 42 cases of osteoblastoma-like osteosarcoma tissues were selected. Samples in ADAM8 group and EGFR group were stained with no detachment. 23 cases of osteochondroma tissues were from patients who aged 5 to 26 There were 13 male and 10 female cases in total, including 3 cases of osteosarcoma on the distal femur and 20 cases of osteosarcoma on the proximal tibia. Besides, there was one case of detachment in ADAM8 group and EGFR group respectively. 35 cases of normal bone tissues were from subjects who aged 58 to 87 . There were 15 male and 20 female cases in total, and all cases were cancellous bones on the neck of femur on the proximal femur. Besides, there were 6 cases of detachment in ADAM8 group and 14 cases in EGFR group. 46 cases of osteoarthritis tissues were from patients who aged 56 to 73 . There were 20 male and 26 female cases in total, and all cases were injured cartilages of osteoarthropathy. Besides, there were 11 cases of detachment in ADAM8 group and 21 cases in EGFR group.

\subsection{Methods}

\subsubsection{Instruments and reagents}

Main reagents contained rabbit anti-human polyclonal antibodies against ADAM8 (AF1031), which were purchased from Shanghai Blue Gene Biotechnology Co., Ltd.; mouse anti-human monoclonal antibodies against EGFR (ZM0083), made by Beijing Zhongshan Golden-bridge Biotechnology Co., Ltd.; Ready-to-use Ultra-sensitive TMS-P kits and DAB substrate kits, which were bought from Fuzhou Maixin Biotechnologies Development Company. Main instruments consisted of one RM2165 Rotary Microtome, one ST5010 Autostainer, one VBP2001 Slide Mate, one Promounter Automatic Sealing Machine, one MTA1 Tissue
Microarrayer, one Excelsior Automatic Dehydration Machine, one Histocentre 3 Embedding Machine and one ESPES Oven.

\subsubsection{Preparation of tissue chips of paraffin-embedded samples}

(1) Processing of tissue samples: extracted and dehydrated tissues first, and then embedded them with paraffin. Paraffin blocks were made into sections and stained with $\mathrm{HE}$; (2) The pathological diagnosis was made to HE-stained sections, with the boundaries of lesion tissues marked; (3) Tissue types and arrangement modes of tissue microarrays were designed according to the research objective, and a proper tissue case number was selected from the tissue database for each tissue microarray; (4) The tissue paraffin block and its corresponding HE-stained section were taken out from the tissue bank according to its tissue case number; (5) Blank receptor paraffin blocks were prepared by the tissue embedding machine; (6) The tissue microarrayer was used to extract tissue chips from pathological paraffin blocks and array them in a highly organized structure on blank receptor paraffin blocks, and the tissue microarray blocks were heated and integrated in the oven at the constant temperature of $52^{\circ} \mathrm{C}$ to make tissue chips tightly attached to receptor paraffin blocks; the automatic microtome was used to trim and process paraffin sections; (7) Tissue microarray sections were placed into the oven at the constant temperature of $60^{\circ} \mathrm{C}$ for $16 \mathrm{~h}$; and then refrigerated in the refrigerator at or under the temperature of $5^{\circ} \mathrm{C}$.

\subsubsection{Immunocytochemical staining}

The conventional immunocytochemical staining was performed to the prepared paraffin-embedded tissue chips.

\subsection{Indicator observation}

Cells in which light yellow, brownish yellow or dark brown particles appeared were considered as positive cells. The semi-quantitative counting method was applied to this research, with three parameters (i.e., staining intensity, the percentage of positive cells and the product of their scores) adopted. Grading standards of staining intensity were as follows: no staining was 0 , light yellow for 1 , brownish yellow for 2 and brown for 3. Five fields of cells were chosen randomly and observed under the light microscope, and the percentage of stained positive cells were recorded accordingly. The grading criteria were as follows: $0 \%$ was 0 , $1 \%-10 \%$ for $1,>10 \%-50 \%$ for $2,>50 \%-80 \%$ for 3 and $>$ $80 \%$ for 4 . The final score was the product of the scores of staining intensity and the percentage of positive cells: 0 indicated negative (-), 1-2 for weakly positive (+), 3-4 for positive $(++)$, and $>4$ for strongly positive $(+++)$. 


\subsection{Statistical treatment}

$\chi^{2}$ test was used to analyze the difference in the expression levels of ADAM8 and EGFR in osteosarcoma tissues, osteochondroma tissues, osteoarthritis tissues and normal bone tissues as well as the relationship of ADAM8 and EGFR expression with tissue pathological types; Spearman's rank correlation test was applied to the correlation analysis. All results were analyzed by SPSS20.0 software, and the difference $p<.05$ was of statistical significance.

\section{RESUlts}

3.1 The expression of ADAM8 and EGFR in osteosarcoma tissues, osteochondroma tissues, osteoarthritis tissues and normal bone tissues

The expression of ADAM8 showed positive results in 34/42 cases of osteoblastoma-like osteosarcoma tissues, with different degrees of staining intensity; and showed negative results in 8 cases, and the positive rate was $80.95 \%$. $8 / 22$ cases of osteochondroma tissues showed a positive expression of ADAM 8 and 14 cases showed a negative expression, and the positive rate was $36.36 \%$. In 35 cases of osteoarthritis tissues, 22 cases showed a positive expression of ADAM8 and 13 cases showed a negative expression, and the positive rate was $62.86 \%$; 7 out of 29 cases of normal bone tissues showed a positive expression and the other 22 cases showed a negative expression, and the positive rate was $24.14 \%$. See Table 1 and Figures 1-4 for details.

\subsection{The expression of EGFR}

The expression of EGFR showed positive results in 28/42 cases of osteoblastoma-like osteosarcoma tissues, with different degrees of staining intensity; and showed negative results in 14 cases, and the positive rate was $62.67 \%$. 2/22 cases of osteochondroma tissues showed a positive expression of EGFR and 20 cases showed a negative expression, and the positive rate was $9.09 \%$. In 25 cases of osteoarthritis tissues, 10 cases showed a positive expression of EGFR and 15 cases showed a negative expression, and the positive rate was $40.00 \%$; 1 out of 21 cases of normal bone tissues showed a positive expression and the other 20 cases showed a negative expression, and the positive rate was $4.67 \%$. See Table 2 and Figures 5-8 for details.

Table 1. The expression of ADAM8

\begin{tabular}{|c|c|c|c|c|}
\hline Tissues type & n & Positive & Negative & Positive Rate (\%) \\
\hline Osteosarcoma Tissues & 42 & 34 & 8 & 80.95 \\
\hline Osteochondroma Tissues & 22 & 8 & 14 & 36.36 \\
\hline Osteoarthritis Tissues & 35 & 22 & 13 & 62.86 \\
\hline Normal Bone Tissues & 29 & 7 & 22 & 24.14 \\
\hline
\end{tabular}

Note. In the comparison among 4 types of tissues, $\chi^{2}=26.591, p=.000<.01$; there was a statistically significant difference in the comparison between osteosarcoma tissues and osteochondroma tissues, osteosarcoma tissues and normal bone tissues, normal bone tissues and osteoarthritis tissues.

Table 2. The expression of EGFR

\begin{tabular}{lllll}
\hline Tissues type & n & Positive (n) & Negative (n) & Positive Rate (\%) \\
\hline Osteosarcoma Tissues & 42 & 28 & 14 & 66.67 \\
Osteochondroma Tissues & 22 & 2 & 20 & 9.09 \\
Osteoarthritis Tissues & 25 & 10 & 15 & 40.00 \\
Normal Bone Tissues & 21 & 1 & 20 & 4.76 \\
\hline
\end{tabular}

Note. In the comparison among 4 types of tissues, $\chi^{2}=32.567, p=.000<.01$; there was a statistically significant difference in the comparison between osteosarcoma tissues and osteochondroma tissues, osteosarcoma tissues and normal bone tissues, normal bone tissues and osteoarthritis tissues.

\subsection{The co-expression of ADAM8 and EGFR in osteosar- coma tissues}

The comprehensive analysis was performed to the staining results of ADAM8 and EGFR in 42 cases of osteoblastomalike osteosarcoma tissues, and Spearman's rank correlation test showed that ADAM8 was positively correlated to EGFR, $r=.379, p=.033<.05$ (see Table 3 ).
Table 3. The co-expression of ADAM8 and EGFR in osteosarcoma tissues

\begin{tabular}{|c|c|c|c|c|}
\hline \multirow{2}{*}{ ADAM8 } & \multicolumn{3}{|c|}{ EGFR } & \multirow{2}{*}{ Total } \\
\hline & ++ & + & - & \\
\hline++ & 5 & 1 & 2 & 8 \\
\hline+ & 6 & 12 & 8 & 26 \\
\hline- & 1 & 3 & 4 & 8 \\
\hline Total & 9 & 19 & 14 & 42 \\
\hline
\end{tabular}




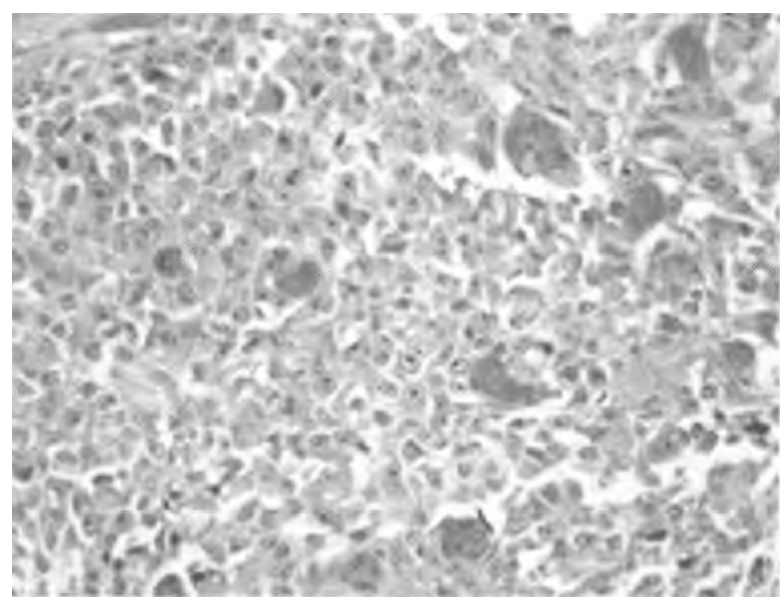

Figure 1. The expression of ADAM8 in osteosarcoma tissues $(++, 200 \times)$

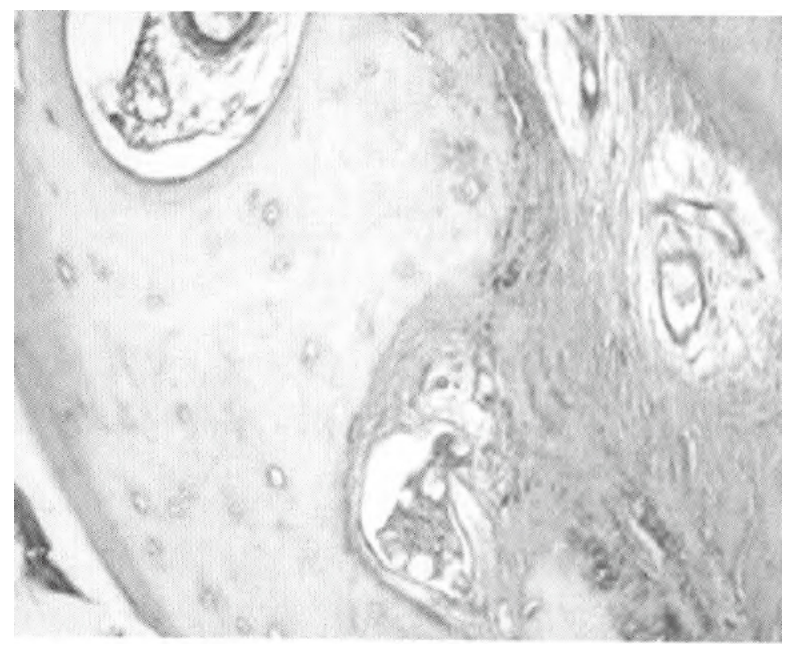

Figure 2. The expression of ADAM8 in osteochondroma tissues $(-, 200 \times)$

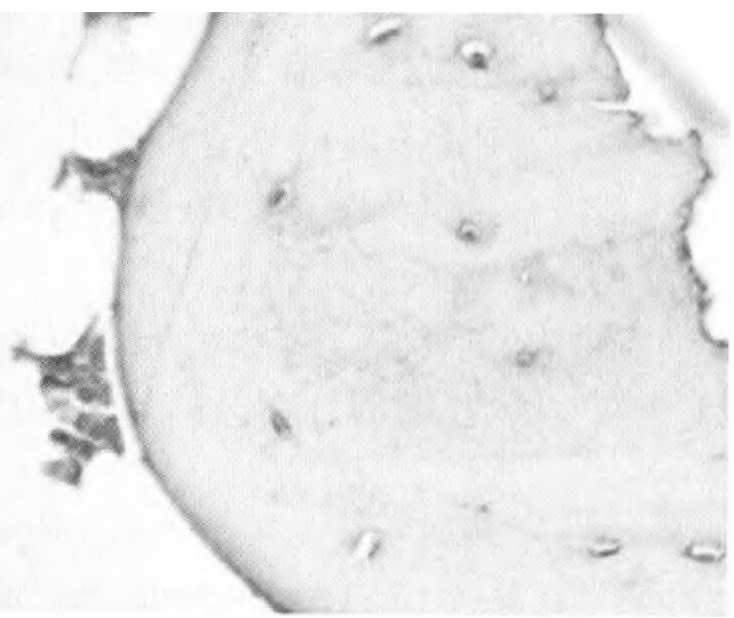

Figure 3. The expression of ADAM8 in normal bone tissues $(-, 200 \times)$

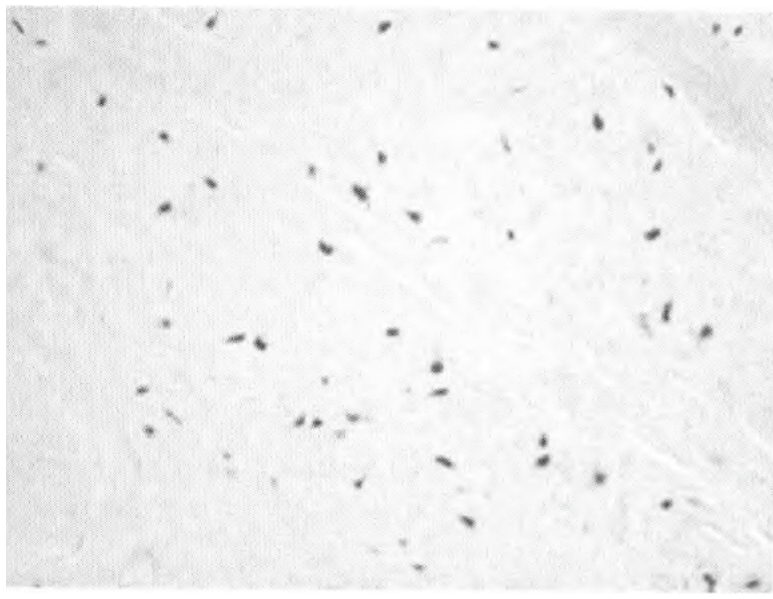

Figure 4. The expression of ADAM8 in osteoarthritis tissues $(+, 200 \times)$

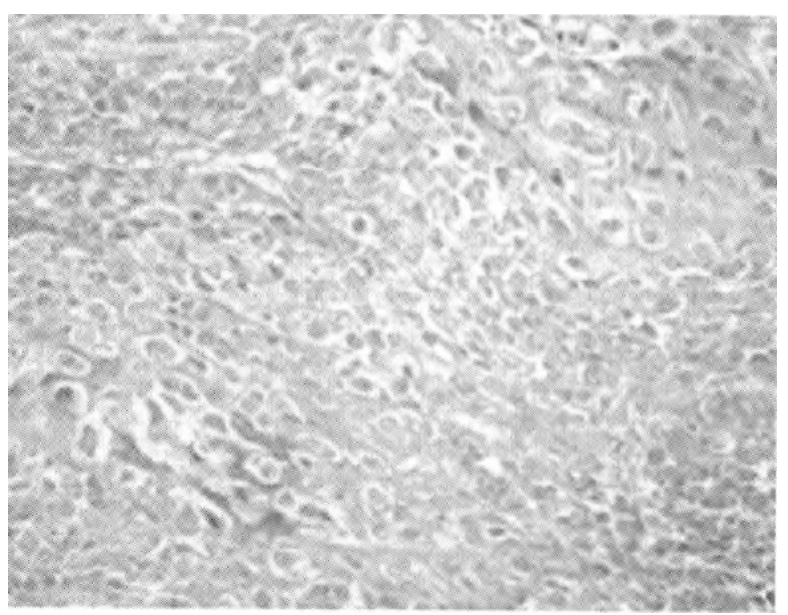

Figure 5. The expression of EGFR in osteosarcoma tissues $(++, 200 \times)$

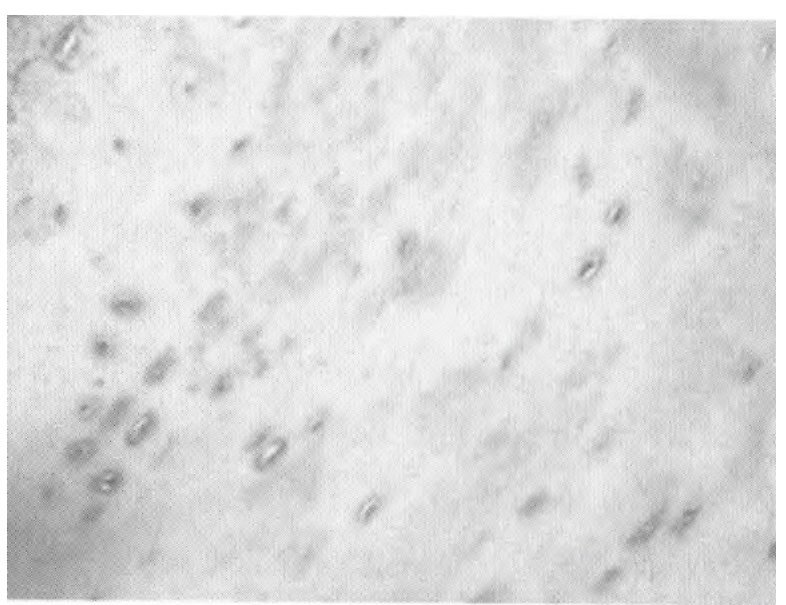

Figure 6. The expression of EGFR in osteochondroma tissues $(-, 200 \times)$ 


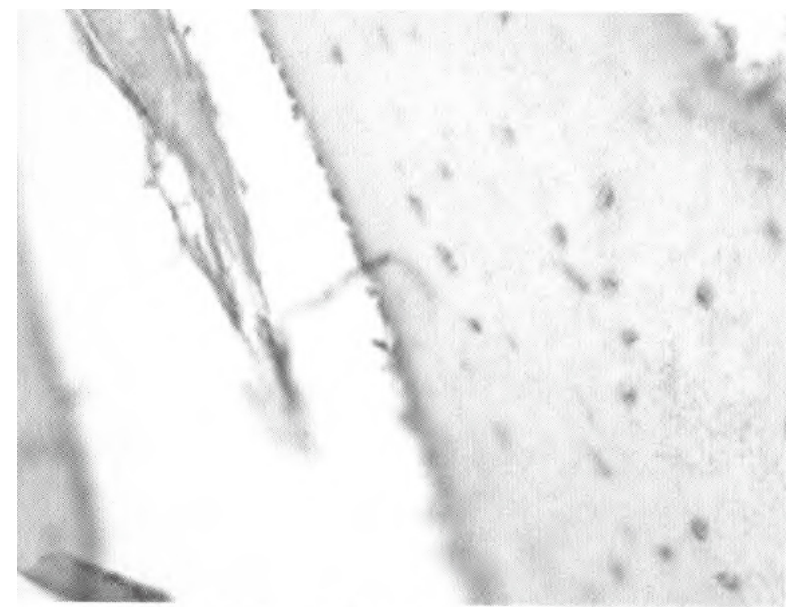

Figure 7. The expression of EGFR in normal bone tissues $(-, 200 \times)$

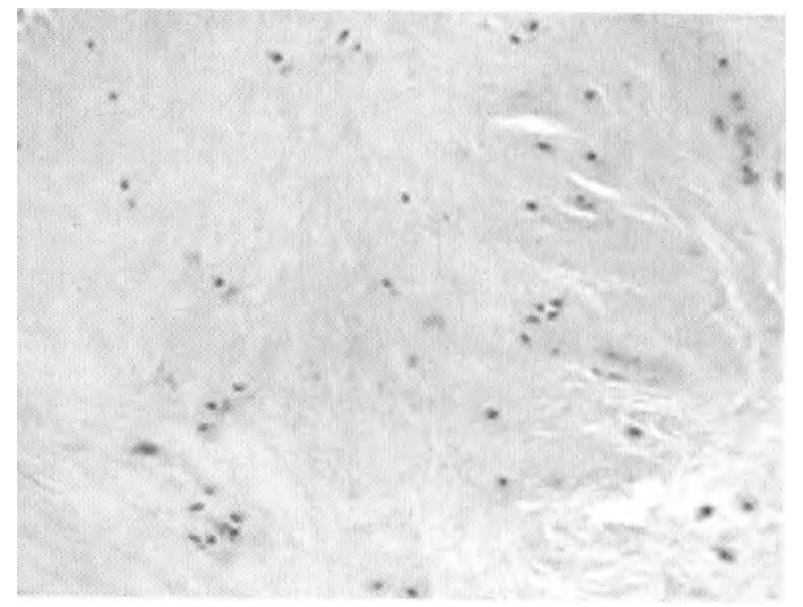

Figure 8. The expression of EGFR in osteoarthritis tissues $(+, 200 \times)$

\section{Discussion}

Osteosarcoma is a type of malignant tumor characterized by osseous or osteoid tissues produced directly by proliferative tumor cells, and it is one of the most frequently seen, rapidlygrowing and primary malignant bone tumors. ADAM8 is a member of ADAMs family, and its cluster of differentiation is CD156. The abnormal expression of ADAM8 has a remarkable physiological significance in some diseases, such as some inflammatory diseases, matrix remodeling of tumor cells, asthma, cerebral injury, bone destruction, neurodegenerative disease and so on. Many scholars have found that the expression of ADAM8 is up-regulated in different types of cancerous tumors. ADAM8 plays a role in the cartilage damage in rheumatoid arthritis, and it may damage cartilages by accelerating osteoclast maturation or hydrolyzing cartilage matrix proteins. There are few related literatures on the expression of ADAM8 in osteosarcoma, benign bone tumors and osseous tissues. The preliminary study of this issue $^{[5,6]}$ analyzes 69 cases of tissue samples from osteosarcoma patients and shows that the up-regulated expression of ADAM8 is related with the prognosis and tissue transfer. In this research, the expression of ADAM8 has been detected in 42 cases of osteoblastoma-like osteosarcoma tissues, 22 cases of osteochondroma tissues, 29 cases of normal bone tissues and 35 cases of osteoarthritis tissues. It is found that the expression of ADAM8 in osteoarthritis tissues is obviously higher than that in normal bone tissues, and the difference is of statistical significance. Combined with relevant references, ADAM8 is speculated to play a role in the inflammatory lesion of articular cartilages and have an effect on the destruction of articular cartilages. Bone destruction is probably related with osteoclast maturation accelerated by ADAM8. It is also found in this research that the expression of ADAM8 in osteosarcoma tissues is significantly higher than that in osteochondroma and normal bone tissues, and the difference is of statistical significance $(p<.05)$. However, there is no statistically significant difference between osteochondroma tissues and normal bone tissues $(p>.05)$. It is indicated that ADAM8 is probably one of factors that affect the development of osteosarcoma. It also plays a role in the course of osteosarcoma by destructing articular cartilages and accelerating osteoclast maturation. ${ }^{[7]}$

Epithelial growth factor receptor is a type of receptor that belongs to ErbB receptor family. EGFR is the expression product of c-erbB-1 and it is one of Type 1 tyrosine kinase growth factor receptors. EGFR can be expressed in epithelial tissues, mesenchyma, neurogenic tissues and vascular tissues. Current researches show that the over-expression of EGFR plays a significant role in the evolution of malignant tumors, for example, the expression of EGFR is up-regulated in neuroglioma, ${ }^{[8]}$ carcinoma of bile duct, colon cancer and carcinoma. EGFR has become one of the most widely concerned, the most intensively studied and the most promising therapeutic targets in the current anti-tumor molecular targeted researches. ${ }^{[9,10]}$ In this research, the expression of EGFR has been detected in 42 cases of osteoblastoma-like osteosarcoma tissues, 22 cases of osteochondroma tissues, 21 cases of normal bone tissues and 25 cases of osteoarthritis tissues. It is found that the expression level of EGFR in osteoarthritis tissues is higher than that in normal bone tissues, and the difference is of statistical significance. It is indicated that EGFR probably participates in the destruction of articular cartilages. However, there is no relevant report concerning whether EGFR can play a regulatory effect on the destruction of articular cartilages. In the future research, it is recommended to increase the number of samples or make a further study in the animal experiment. It is also found in 
this research that the expression of EGFR in osteosarcoma tissues is significantly higher than that in osteochondroma and normal bone tissues, and the difference is of statistical significance. However, there is no statistically significant difference between osteochondroma tissues and normal bone tissues. The experimental results are consistent with relevant literatures, which indicate that both EGFR and ADAM8 are regulatory factors in the development of osteosarcoma. ${ }^{[1]}$

That ADAM8 can accelerate osteoclast maturation and destruct the function of articular cartilages is speculated to be one of factors that cause the bone destruction peripheral to osteosarcoma tissues. One of osteosarcoma proliferation and invasion mechanisms is to affect the binding of EGFR to its ligand and then activate EGFR-mediated signaling pathway through the detachment of ADAM8 extracellular domain.

The shortcoming of this research is that these two factors are only studied and analyzed morphologically, however, the difference in their expression in histological subtypes of osteosarcoma remains to be studied. In the future research, it is recommended to observe the expression of ADAM8 and
EGFR in osteosarcoma cell lines, with the help of stimuli and/or inhibitors, to further study the expression mechanism of these two factors and their correlation. The genetic technology can also be used to silence the gene expression of ADAM8 and EGFR in osteosarcoma cells and then block ADAM8- and EGFR-mediated malignant biological behaviors by synthesizing chemically siRNA in vitro and construct plasmid vectors, so as to provide a new strategy for the therapy of osteosarcoma.

\section{Conclusion}

There is no relevant report on the co-expression of ADAM8 and EGFR in osteosarcoma tissues. This research analyzes the expression of ADAM8 and EGRF in 42 cases of osteoblastoma-like osteosarcoma tissues by use of tissue chips combined with immunohistochemistry, and finds that the expression of ADAM8 is correlated to the expression of EGFR in osteosarcoma tissues through statistical calculation.

\section{Conflicts of InTEREST Disclosure}

The authors declare they have no conflicts of interest.

\section{REFERENCES}

[1] Yao Y, Dong Y, Lin F, et al. The expression of CRM1 is associated with prognosis in human osteosarcoma. Oncol Rep. 2009; 21(1): 229-235.

[2] Jaffe N. Adjuvant chemotherapy in osteosarcoma: an odyssey of rejection and vindication. Cancer Treat Res. 2009; 152(152): 219. PMid:20213393. https : //doi .org/10.1007/978-1-441 9-0284-9_11

[3] Dukstra A, Postma D S, Noordhoek J A, et al. Expression of ADAMs (a disintegrin and metalloprotease) in the human lung. Virchows Arch. 2009; 454(4): 441-449. PMid:19255780. https: //doi.org/10.1007/s00428-009-0748-4

[4] Hernandez I, Moreno J L, Zandueta C, et al. Novel alternatively spliced ADAM8 isoforms contribute to the aggressive bone metastatic phenotype of lung cancer. Oncogene. 2010; 29(26): 3758-3769. PMid:20453887. https ://doi .org/10.1038/onc.2010.130

[5] Li ZH, Liao QD, Wu YC, et al. Upregulation of a disintegrin and metalloprotease 8 influences tumor metastasis and prognosis in patients with osteosarcoma. Pathol Oncol Res. 2012; 18(3): $657-$ 661. PMid:22215309. https : //doi.org/10.1007/s12253-011 $-9491-7$
[6] Ishizuka H, Garca-Palacios V, Lu G, et al. ADAM8 enhances osteoclast precursor fusion and osteoclast formation in vitro and in vivo. J Bone Miner Res. 2012; 26(1): 169-181. PMid:20683884. https ://doi.org/10.1002/jbmr.199

[7] Jianshu W, Zhigang Y, Yanchuan P, et al. RK5 and MMP-9 expression levels in osteosarcoma and their clinical significance. Chinese Journal of Clinical Oncology. 2017; 44(14): 689-694.

[8] Xuemei M, Shifeng Y, Min L, et al. Expression of macrophage inflammatory protein- $1 \alpha$, a disintegrin-like and metalloproteinase 8 and 12, and CD68 protein in giant cell lesions of jaw and giant cell tumors of long bone. Chinese Journal of Pathology. 2005; 34(7): 393-396.

[9] Ying L, Cuiping R, Jingliang C, et al. Contrastive analysis on the DWI, MRS and DCE results in nuclear magnetic resonance examinations in patients with low and highly malignant primary osteosarcoma. Shandong Medical Journal. 2017; 57(23): 80-83.

[10] Xin X, Zhen W, Pin X, et al. Gene study and individualized therapy of osteosarcoma on the basis of High-throughput sequencing. Chinese Journal of Orthopaedics. 2014; 34(4): 466-471.

[11] Zhenhua H, Futing Z, Qiong D, et al. Clinical analysis on 60 cases of non-classical high-grade osteosarcoma. Guangdong Medical Journal. 2017; 38(16): 2506-2510. 